UT-780

\title{
Dynamical Tuning of the Initial Condition for New Inflation in Supergravity
}

\author{
Izawa K.-I. $^{a}$, M. Kawasaki ${ }^{b}$ and T. Yanagida ${ }^{a}$ \\ ${ }^{a}$ Department of Physics, University of Tokyo, Tokyo 133, Japan \\ ${ }^{b}$ Institute for Cosmic Ray Research, University of Tokyo, Tokyo 188, Japan
}

July, 1997

\begin{abstract}
We point out that for a class of 'new inflation' models in supergravity the required initial value of the inflaton field is dynamically set if there is another inflation ('pre-inflation') before the 'new inflation'. We study the dynamics of both inflatons by taking a hybrid inflation model for the 'pre-inflation' as an example. We find out that our 'new inflation' model provides reheating temperatures $T_{R} \simeq 10 \mathrm{MeV}-10^{4} \mathrm{GeV}$ low enough to avoid the gravitino problem even in gauge-mediated supersymmetry-breaking models. We also construct a model where the scale for the 'new inflation' is generated by nonperturbative dynamics of a supersymmetric gauge theory.
\end{abstract}




\section{Introduction}

The hypothesis of an exponentially expanding (inflationary) stage in the early universe is a very attractive idea, since it solves the flatness and the horizon problems [1] in the standard cosmology. There have been proposed various models to realize the inflationary epoch in the early universe [2]. Among them the 'new inflation' model [3] is the most interesting in the point that its reheating temperature $T_{R}$ is expected to be sufficiently low to avoid the overproduction of gravitinos in supergravity [4. In particular, the gauge-mediated supersymmetry(SUSY)-breaking model [5, 6, 7] predicts the mass of gravitino $m_{3 / 2}$ in a range of $10^{2} \mathrm{keV}-1 \mathrm{GeV}[8]$. In this case the upper bounds of the reheating temperature should be $T_{R}<10^{2} \mathrm{GeV}-10^{6} \mathrm{GeV}$ for $m_{3 / 2} \simeq 10^{2} \mathrm{keV}-1 \mathrm{GeV}$ [9] This constraint is easily satisfied in a large class of 'new inflation' models.

However, the 'new inflation' model has a serious drawback. It requires an extreme fine tuning of the initial condition [2] to have a sufficiently long inflation: the universe has to have a large region over horizons at the beginning where the inflation field $\varphi$ is smooth and its average value is very close to a local maximum of the potential $V(\varphi)$. Since the inflaton potential $V(\varphi)$ should be very flat to satisfy the slow-roll condition, there is no dynamical reason for the universe to choose such a specific initial value of the $\varphi$.

In this paper we point out that if there exists another inflation ('pre-inflation') before the 'new inflation', the required initial condition for the 'new inflaton' $\varphi$ is

\footnotetext{
${ }^{1}$ If $m_{3 / 2} \lesssim 1 \mathrm{keV}$, we have no constraint on the reheating temperature. There have been found a few models [10, 11 accommodating such a light gravitino, so far.
} 
dynamically realized owing to supergravity effects.t] To demonstrate our point, we give an explicit model for both of the 'new inflation' and the 'pre-inflation'. We also construct a dynamical model which produces the desired potential for 'new inflation'.

\section{A 'new inflation' model}

Our model consists of two inflaton potentials: one is for a 'new inflation' and the other for a 'pre-inflation'. In this section we consider the part of the 'new inflation'. We adopt a 'new inflation' model proposed recently in Ref. [12], which is based on an $R$ symmetry in supergravity.

The inflaton superfield $\phi(x, \theta)$ is assumed to have an $R$ charge $2 /(n+1)$ so that the following tree-level superpotential is allowed:

$$
W_{0}(\phi)=-\frac{g}{n+1} \phi^{n+1}
$$

where $n$ is a positive integer and $g$ denotes a coupling constant of order one. Here and hereafter, we set the gravitational scale $M \simeq 2.4 \times 10^{18} \mathrm{GeV}$ equal to unity and regard it as a plausible cutoff in supergravity. We further assume that the continuous $U(1)_{R}$ symmetry is dynamically broken down to a discrete $Z_{2 n R}$ at a scale $v$ generating an effective superpotential [12, 13]:

$$
W_{e f f}(\phi)=v^{2} \phi-\frac{g}{n+1} \phi^{n+1} .
$$

\footnotetext{
${ }^{2}$ In a large class of 'new inflation' models the Hubble parameter is much smaller than the gravitational scale. Thus, the 'new inflation' itself does not give a perfect explanation for why our universe lived for such a long time. To solve this problem the presence of the 'pre-inflation' with a large Hubble parameter is desirable [2].
} 
An explicit dynamics to induce this superpotential will be given in section 4 .

The $R$-invariant effective Kähler potential is given by

$$
K(\phi)=|\phi|^{2}+\frac{k}{4}|\phi|^{4}+\cdots
$$

where $k$ is a constant of order one.

The effective potential for a scalar component of the superfield $\phi(x, \theta)$ in supergravity is given by [14]

$$
V=e^{K(\phi)}\left\{\left(\frac{\partial K}{\partial \phi \partial \phi^{*}}\right)^{-1}\left|D_{\phi} W\right|^{2}-3|W|^{2}\right\},
$$

with

$$
D_{\phi} W=\frac{\partial W}{\partial \phi}+\frac{\partial K}{\partial \phi} W
$$

where $\phi(x)$ denotes a scalar component of $\phi(x, \theta)$. This potential yields a vacuum

$$
\langle\phi\rangle \simeq\left(\frac{v^{2}}{g}\right)^{\frac{1}{n}}
$$

in which we have a negative energy as

$$
\langle V\rangle \simeq-3 e^{\langle K\rangle}|\langle W\rangle|^{2} \simeq-3\left(\frac{n}{n+1}\right)^{2}|v|^{4}|\langle\phi\rangle|^{2}
$$

It is a very interesting assumption in Ref. [12] that the negative vacuum energy (7) is canceled out by a SUSY-breaking effect which gives a positive contribution $\Lambda_{S U S Y}^{4}$ to the vacuum energy. Namely, we impose

$$
-3\left(\frac{n}{n+1}\right)^{2}|v|^{4}\left|\frac{v^{2}}{g}\right|^{\frac{2}{n}}+\Lambda_{S U S Y}^{4}=0 .
$$

In supergravity the gravitino acquires a mass

$$
m_{3 / 2} \simeq \frac{\Lambda_{S U S Y}^{2}}{\sqrt{3}}=\left(\frac{n}{n+1}\right)|v|^{2}\left|\frac{v^{2}}{g}\right|^{\frac{1}{n}} .
$$


The inflaton $\phi$ has a mass $m_{\phi}$ in the vacuum with

$$
m_{\phi} \simeq n|g|^{\frac{1}{n}}|v|^{2-\frac{2}{n}} .
$$

The inflaton $\phi$ may decay into the ordinary particles through $R$-invariant interactions with the ordinary light fields $\psi_{i}$ in the Kähler potential. In general, we have the following interactions:

$$
K\left(\phi, \psi_{i}\right)=\sum k_{i}|\phi|^{2}\left|\psi_{i}\right|^{2} .
$$

With these interactions the reheating temperature is given by (see Ref. [12] for details)

$$
T_{R} \simeq 0.1 n^{\frac{3}{2}}|g|^{\frac{2}{n+1}} m_{3 / 2}^{\frac{3 n-1}{2(n+1)}},
$$

for $k_{i} \simeq 1$. Requiring $T_{R} \gtrsim 10 \mathrm{MeV}$, we get a constraint on $m_{3 / 2}$ :

$$
m_{3 / 2} \gtrsim n^{-\frac{n+1}{3 n-1}}|g|^{\frac{4}{3 n-1}} 10^{\frac{39(n+1)}{3 n-1}} .
$$

For $g \simeq 1$ and $n=4$, we have

$$
m_{3 / 2} \gtrsim 10^{-18}(\simeq 3 \mathrm{GeV}) .
$$

This already seems to exclude the gauge-mediated SUSY-breaking model in the present scenario. However, if a pair of Higgs doublets $H$ and $\bar{H}$ in the SUSY standard model has a suitable $U(1)_{R}$ charge, we may have a Kähler interaction with

$$
K(\phi, H, \bar{H})=h \phi^{*} H \bar{H}+\text { h.c. }
$$

Then we obtain the reheating temperature

$$
T_{R} \simeq 0.1 m_{\phi}^{3 / 2} \simeq 0.1 n^{\frac{3}{2}}\left(\frac{n+1}{n}\right)^{\frac{3(n-1)}{2(n+1)}}|g|^{\frac{3\left(n^{2}+1\right)}{2 n(n+1)}} m_{3 / 2}^{\frac{3(n-1)}{2(n+1)}} .
$$

\footnotetext{
${ }^{3}$ It has been shown in Ref. 12 that $n=4$ is the most plausible case.
} 
The requirement $T_{R} \gtrsim 10 \mathrm{MeV}$ leads to

$$
m_{3 / 2} \gtrsim 3 \times 10^{-23}(\simeq 0.07 \mathrm{MeV}),
$$

for $g \simeq 1$ and $n=4$. We assume this case in this paper since it accommodates the light gravitino $\left(m_{3 / 2} \lesssim 1 \mathrm{GeV}\right)$ predicted in the gauge-mediated SUSY-breaking model.f

Let us now discuss the inflationary dynamics of our 'new inflation' model. We identify the inflaton field $\varphi(x) / \sqrt{2}(\geq 0)$ with the real part of the field $\phi(x)$. The potential for the inflaton is given by

$$
V(\varphi) \simeq v^{4}-\frac{k}{2} v^{4} \varphi^{2}-\frac{g}{2^{\frac{n}{2}-1}} v^{2} \varphi^{n}+\frac{g^{2}}{2^{n}} \varphi^{2 n},
$$

for $\varphi\langle\langle\varphi\rangle=\sqrt{2}\langle\phi\rangle$. Here, $g$ and $v$ are taken to be positive. Notice that the $k$-independent contribution of $\varphi^{2}$ term in $e^{K}\left|D_{\phi} W\right|^{2}$ is exactly canceled by that in $-3|W|^{2}$ as stressed in Ref. [13, [16].

It has been shown in Ref. [12] that the slow-roll condition for the inflaton is satisfied for $k<1$ and $\varphi \lesssim \varphi_{f}$ where

$$
\varphi_{f} \simeq \sqrt{2}\left(\frac{(1-k) v^{2}}{g n(n-1)}\right)^{\frac{1}{n-2}} .
$$

This provides the value of $\varphi$ at the end of inflation. The Hubble parameter during the inflation $\left(0<\varphi \lesssim \varphi_{f}\right)$ is given by

$$
H \simeq \frac{\sqrt{V}}{\sqrt{3}} \simeq \frac{v^{2}}{\sqrt{3}} .
$$

As shown in Ref. [12], the spectral index $n_{s}$ of the density fluctuations is given by

$$
n_{s} \simeq 1-2 k \text {. }
$$

\footnotetext{
${ }^{4}$ As mentioned in the introduction, we do not have any cosmological constraints if $m_{3 / 2} \lesssim 1 \mathrm{keV}[15$.
} 
By using the experimental constraint $n_{s} \gtrsim 0.6$ [17, [18], we take $k \lesssim 0.2$. Since there is no symmetry reason to suppress the $k$ term, we assume $k \gtrsim 0.02$. With $0.02 \lesssim k \lesssim 0.2$, we obtain the $e$-fold number $N$ as

$$
\begin{aligned}
N & \simeq \int_{\tilde{\varphi}}^{\varphi_{N}} d \varphi \frac{v^{4}}{-k v^{4} \varphi}+\int_{\varphi_{f}}^{\tilde{\varphi}} d \varphi \frac{v^{4}}{-n g 2^{-\frac{n-2}{2}} v^{4} \varphi^{n-1}} \\
& =\frac{1}{k} \ln \left(\frac{\tilde{\varphi}}{\varphi_{N}}\right)+\frac{1-n k}{(n-2) k(1-k)},
\end{aligned}
$$

where

$$
\begin{aligned}
\varphi_{N} & \simeq \tilde{\varphi} e^{-k \bar{N}} \\
\tilde{\varphi} & =\sqrt{2}\left(\frac{k v^{2}}{g n}\right)^{\frac{1}{n-2}}, \\
\bar{N} & =N-\frac{1-n k}{(n-2) k(1-k)} .
\end{aligned}
$$

We use the $e$-fold number $N$ of the present horizon [2]

$$
\begin{aligned}
N & \simeq 66+\ln (H)+\frac{1}{3} \ln \left(T_{R}\right)-\frac{2}{3} \ln \left(m_{\varphi}\right)-\frac{2}{3} \ln (\langle\varphi\rangle) \\
& \simeq 50
\end{aligned}
$$

to get $\varphi_{N} \simeq\left(10^{-5}-0.1\right) v$ for $0.02 \lesssim k \lesssim 0.2, g \simeq 1$ and $n=4$.

The amplitude of primordial density fluctuations $\delta \rho / \rho$ is given by

$$
\frac{\delta \rho}{\rho} \simeq \frac{1}{5 \sqrt{3} \pi} \frac{V^{3 / 2}\left(\varphi_{N}\right)}{\left|V^{\prime}\left(\varphi_{N}\right)\right|}=\frac{1}{5 \sqrt{3} \pi} \frac{v^{2}}{k \varphi_{N}} .
$$

From the COBE normalization $[18$

$$
\frac{V^{3 / 2}\left(\varphi_{N}\right)}{\left|V^{\prime}\left(\varphi_{N}\right)\right|} \simeq 5.3 \times 10^{-4},
$$

We obtain

$$
v \simeq 3.7 \times 10^{-4} k^{3 / 2} g^{-1 / 2} e^{-k \bar{N}}
$$




$$
\begin{aligned}
& =1.8 \times 10^{-9}-6.8 \times 10^{-7} \\
m_{3 / 2} & \simeq 2.7 \times 10^{-9} k^{15 / 4} g^{-3 / 2} e^{-5 k \bar{N} / 2} \\
& =0.35 \mathrm{MeV}-9.3 \times 10^{2} \mathrm{GeV},
\end{aligned}
$$

for $0.02 \lesssim k \lesssim 0.2$ and $g \simeq 1$. Here we have assumed $n=4$.

We find that our 'new inflation' model can accommodate the gravitino in a large range of masses $m_{3 / 2} \simeq 0.3 \mathrm{MeV}-1 \mathrm{TeV}$ with the reheating temperature $T_{R} \simeq$ $40 \mathrm{MeV}-2 \times 10^{4} \mathrm{GeV}$ and $n_{s} \simeq 0.6-0.96$. initial value of $\varphi \lesssim \varphi_{N} \simeq\left(10^{-5}-0.1\right) v$, so far. In the next section we show that another inflation ('pre-inflation') before the 'new inflation' naturally sets the initial value of the $\varphi \lesssim \varphi_{N}$ through supergravity effects.

\section{A 'pre-inflation' model}

In this section we discuss another inflation before the 'new inflation' and show that the initial value required for the $\varphi(x)$ is dynamically tuned during the 'pre-inflation'. We adopt a hybrid inflation model in Ref. [19] as an example of the 'pre-inflation'.

The hybrid inflation model contains two kinds of superfields: one is $S(x, \theta)$ and the others are $\Psi(x, \theta)$ and $\bar{\Psi}(x, \theta)$. The model is also based on the $U(1)_{R}$ symmetry. The superpotential is given by [16, 19]

$$
W=-\mu^{2} S+\lambda S \bar{\Psi} \Psi
$$

\footnotetext{
${ }^{5}$ If one imposes $m_{3 / 2} \lesssim 1 \mathrm{GeV}$ as suggested from the gauge-mediated SUSY-breaking model 5,6 , [], one predicts $n_{s} \lesssim 0.75$, which may be tested in future observations on the microwave background radiation.

${ }^{6}$ Symmetries of this model are discussed in Ref. [16, 19].
} 
The $R$-invariant Kähler potential is given by

$$
K(S, \Psi, \bar{\Psi})=|S|^{2}+|\Psi|^{2}+|\bar{\Psi}|^{2}-\frac{k^{\prime}}{4}|S|^{4}+\cdots
$$

where the ellipsis denotes higher-order terms, which we neglect in the present analysis.

The potential in supergravity is given by

$$
V \simeq\left|\mu^{2}-\lambda \bar{\Psi} \Psi\right|^{2}+|\lambda \Psi S|^{2}+|\lambda \bar{\Psi} S|^{2}+k^{\prime} \mu^{4}|S|^{2}+\cdots
$$

where scalar components of the superfields are denoted by the same symbols as the corresponding superfields. We readily see that if the universe starts with sufficiently large value of $S$, the inflation occurs for $0<k^{\prime}<1$ and continues until $S \simeq S_{c}=$ $\mu / \sqrt{\lambda}$. The inflaton field $\sigma / \sqrt{2}$ is identified with the real part of $S(x)$. The potential is written as

$$
V \simeq\left|\mu^{2}-\lambda \bar{\Psi} \Psi\right|^{2}+\frac{|\lambda|^{2}}{2} \sigma^{2}\left(|\Psi|^{2}+|\bar{\Psi}|^{2}\right)+\frac{k^{\prime}}{2} \mu^{4} \sigma^{2}+\cdots
$$

The Hubble parameter and $e$-folding factor $N^{\prime}$ are given by

$$
\begin{aligned}
H & \simeq \frac{\mu^{2}}{\sqrt{3}} \\
N^{\prime} & \simeq \frac{1}{k^{\prime}} \ln \frac{\lambda \sigma_{i}^{2}}{2 \mu^{2}} .
\end{aligned}
$$

The point is that the 'pre-inflation' implies a dynamical tuning of the initial condition for the 'new inflation' in supergravity. During the 'pre-inflation' the inflaton field $\varphi(x)$ that causes the 'new inflation' gets an effective mass $\mu^{2}$ from the $e^{K} V$ term [20]. Since this effective mass $(\simeq \sqrt{3} H)$ is larger than the Hubble parameter during the 'pre-inflation', the $\varphi$ oscillates with its amplitude decreasing as $a^{-3 / 2}$ 
where $a$ denotes the scale factor. Thus, at the end of the 'pre-inflation', the $\varphi$ takes a value

$$
\varphi \simeq \varphi_{i} e^{-\frac{3}{2} N^{\prime}}
$$

where $\varphi_{i}$ is the value of $\varphi$ at the beginning of the 'pre-inflation'. We take $\varphi_{i} \sim 1$. Requiring $\varphi \lesssim \varphi_{N}$, we get

$$
N^{\prime} \gtrsim 10-20
$$

for $n=4, g \simeq 1$ and $k=0.02-0.2$. Eq.(38) suggests $\sigma_{i} \gtrsim(3.8-10) \mu / \sqrt{\lambda}$ for $k^{\prime} \simeq 0.1$, which is consistent with the assumption $\sigma_{i} \lesssim 1$ even for $\mu \simeq 10^{-2}$. I

However, the above condition is not sufficient since the minimum of the potential for $\varphi$ deviates from zero through the effect of $\left|D_{S} W\right|^{2}+\left|D_{\phi} W\right|^{2}-3|W|^{2}$ term. The effective potential for $\varphi$ during the 'pre-inflation' is written as 8

$$
V(\varphi) \simeq \frac{1}{2} \mu^{4} \varphi^{2}+\frac{\sqrt{2}}{\sqrt{\lambda}} v^{2} \mu^{3} \varphi+\cdots .
$$

This potential has a minimum

$$
\varphi_{\min } \simeq-\frac{\sqrt{2}}{\sqrt{\lambda}} v\left(\frac{v}{\mu}\right) .
$$

For a successful 'new inflation' this minimum should be less than $\varphi_{N}$. Therefore, we obtain the constrain on $\mu$ as $\mu \gtrsim\left(10^{2}-10^{6}\right) v$ for $\lambda \simeq 0.1$.

So far, we have seen that if $\mu \gtrsim\left(10^{2}-10^{6}\right) v$ and $\sigma_{i} \gtrsim(12-32) \mu$ for $\lambda \simeq 0.1$ and $k^{\prime} \simeq 0.1$, the inflaton field $\varphi$ for the 'new inflation' takes a value consistent with the initial condition for the 'new inflation' at the end of the 'pre-inflation'.

\footnotetext{
${ }^{7}$ As pointed out in Ref. [21], one-loop correction to the potential is sizable if the coupling $\lambda$ is large $\left(\lambda \gtrsim 4.3 k^{\prime 1 / 3} \mu^{2 / 3}\right)$. When the one-loop correction controls the slow-roll dynamics, the condition $N^{\prime} \gtrsim 10-20$ leads to $\sigma_{i} \gtrsim(0.5-0.7) \lambda$, which is still consistent with $\sigma_{i} \lesssim 1$ as far as $\lambda \lesssim 1$

${ }^{8}$ For simplicity, we neglect the $|\phi|^{2}|S|^{2}$ term in the Kähler potential.
} 
We now discuss quantum effects during the 'pre-inflation'. It is known that in the de Sitter universe massless fields have quantum fluctuations whose amplitude is given by $H /(2 \pi)$. If the fluctuations of the inflaton $\varphi$ was larger than $\sim\left(10^{-5}-0.1\right) v$, we would yet have the initial value problem. Fortunately, since the mass of the $\varphi$ during the 'pre-inflation' is not less than the Hubble parameter, the quantum fluctuations for $\varphi$ are strongly suppressed [22]. In fact, the quantum fluctuations for $\varphi$ with wavelength corresponding to the horizon at the beginning of the 'new inflation' are given by $\delta \varphi \simeq(H / 2 \pi) \exp \left[-(3 / 2)\left(N^{\prime}-\ln (\mu / v)\right)\right]$. Requiring $\delta \varphi \lesssim\left(10^{-5}-0.1\right) v$ and using Eq.(36) we obtain a constraint, $\mu \lesssim(4-9) \times 10^{-2}$, for $k^{\prime} \simeq 0.1, \lambda \simeq 0.1$ and $\sigma_{i} \lesssim 1$. Therefore, the dynamical tuning of the initial value of $\varphi$ discussed in this section does work even if the Hubble parameter $H=\mu^{2} / \sqrt{3}$ is larger than $v$.

\section{A dynamical model generating the potential for the 'new inflation'}

We have two independent scales $v \simeq 10^{-9}-10^{-6}$ and $10^{-3}-10^{-4} \lesssim \mu \lesssim 4 \times 10^{-2}-$ $9 \times 10^{-2}$ as the scales of the 'new inflation' and the 'pre-inflation', respectively. We may identify the scale of the 'pre-inflation' with that of grand unification, $\mu \simeq 10^{-2}$, which may be related to some fundamental physics in supergravity. On the other hand, it is very natural to consider that the scale of the 'new inflation' arises from nonperturbative dynamics of a SUSY gauge theory, since it is so small compared with the gravitational scale. In this section we construct a model where the scale of the 'new inflation' has the dynamical origin. We take $n=4$ for simplicity. Its generalization is manifest. 
Our model is based on a SUSY $S U(2)$ gauge theory with four doublet hyperqaurks $Q_{i}^{\alpha}(\alpha=1,2, i=1, \cdots, 4)$. An effective superpotential [23] which describes the dynamics of the $S U(2)$ gauge interaction may be given by

$$
W_{e f f}=X\left(\operatorname{Pf} V_{i j}-\Lambda^{4}\right)
$$

in terms of gauge-invariant degrees of freedom

$$
V_{i j} \sim Q_{i} Q_{j}
$$

where the $X$ is an additional chiral superfield and Pf denotes the Pfaffian. The global $S U(4)$ flavor symmetry is spontaneously broken and massless Nambu-Goldstone fields are produced. To avoid such massless fields, we explicitly break the global $S U(4)$ down to $S P(4)$ by introducing an antisymmetric singlet superfield $Y^{i j}(=$ $-Y^{j i}$ ) which constitutes 5 of the global $S P(4)$. Then we may have an $S P(4)$ invariant superpotential

$$
W=h Y^{i j} Q_{i} Q_{j}
$$

Together with the superpotential Eq.(41), we have a unique $S P(4)$-invariant vacuum

$$
\left\langle Q_{1} Q_{2}\right\rangle=\left\langle Q_{3} Q_{4}\right\rangle=\langle(Q Q)\rangle=\Lambda^{2} ; \quad(Q Q) \equiv \frac{1}{2}\left(Q_{1} Q_{2}+Q_{3} Q_{4}\right)
$$

We now introduce a singlet superfield $\phi(x, \theta)$. We assume the $U(1)_{R}$ charges of $Q^{i}$ and $\phi$ to be $2 / 5$. Then we obtain a $U(1)_{R}$-invariant superpotential'

$$
W(Q, \phi)=g^{\prime}(Q Q)^{2} \phi-\frac{g}{5} \phi^{5}
$$

where we have imposed a discrete symmetry $Q Q \rightarrow-Q Q$ and $\phi \rightarrow \phi$. The scale of the 'new inflation' considered in section 2 is given by

$$
v^{2}=g^{\prime} \Lambda^{4}
$$

\footnotetext{
${ }^{9}$ In the limit of $g=0$, the SUSY is dynamically broken 24.
} 
For $v \simeq 10^{-9}-10^{-6}$ and $g^{\prime} \simeq 1$ we obtain $\Lambda \simeq 10^{-5}-10^{-3}\left(\simeq 10^{13}-10^{15} \mathrm{GeV}\right)$.

\section{Conclusion}

The 'new inflation' model is the most attractive one among various inflation models in the point that it provides a reheating temperature low enough to avoid the overproduction of gravitinos. However, the 'new inflation' requires an extreme fine tuning of the initial value of the inflaton field $\varphi$. In this paper we have pointed out that for a class of 'new inflation' models $\square$ the required initial value of the $\varphi$ is dynamically set if there is another inflation before the 'new inflation'.

Although we have used a hybrid inflation model for the 'pre-inflation' as an example, our observation is more general. Any inflation that occurs before the 'new inflation' can tune the necessary initial value of the inflaton $\varphi(\varphi \simeq 0)$ for the 'new inflation' through the supergravity effects if the scale of 'pre-inflation' is sufficiently large.

\footnotetext{
${ }^{10}$ It may be remarkable that the origin $\varphi=0$ is a unique $Z_{2 n R}$-invariant point in the "new inflation' model in Ref. 12. A model with a similar symmetry has been recently proposed in Ref. [25].
} 


\section{References}

[1] A.H. Guth, Phys. Rev. D23, 347 (1981);

K. Sato, Mon. Not. R. astr. Soc. 195, 467 (1981).

[2] For example, A.D. Linde, Particle Physics and Inflationary Cosmology, (Harwood, Chur, Switzerland, 1990).

[3] A. Albrecht and P.J. Steinhardt, Phys. Rev. Lett. 48 (1982) 1220;

A.D. Linde, Phys. Lett. B108 (1982) 389.

[4] M. Yu. Khlopov and A.D. Linde, Phys. Lett. B138 (1984) 265;

J. Ellis, E. Kim and D.V. Nanopoulos, Phys. Lett. B145 (1984) 181;

J. Ellis, G.B. Gelmini, J.L. Lopez, D.V. Nanopoulos and S. Sarker, Nucl. Phys. B373 (1992) 399;

M. Kawasaki and T. Moroi, Prog. Theor. Phys. 93 (1995) 879 hep-ph/9403364.

[5] M. Dine and A.E. Nelson, Phys. Rev. D48 (1993) 1277;

M. Dine, A.E. Nelson and Y. Shirman, Phys. Rev. D51 (1995) 1362;

M. Dine, A.E. Nelson, Y. Nir and Y. Shirman, Phys. Rev. D53 (1996) 2658.

[6] T. Hotta, Izawa K.-I. and T. Yanagida, Phys. Rev. D55 (1996) 415 hepph/9606203.

[7] E. Poppitz and S. Trivedi, hep-ph/9609529;

N. Haba, N. Maru and T. Matsuoka, hep-ph/9612468, hep-ph/9703250;

N. Arkani-Hamed, J. March-Russel and H. Murayama, hep-ph/9701286;

Y. Shadmi, hep-ph/9703312;

H. Murayama, hep-ph/9705271. 
[8] A. de Gouvêa, T. Moroi and H. Murayama, hep-ph/9701244.

[9] T. Moroi, H. Murayama and M. Yamaguchi, Phys. Lett. B303 (1993) 289.

[10] Izawa K.-I., hep-ph/9704382.

[11] Izawa K.-I., D. Nomura, K. Tobe and T. Yanagida, hep-ph/9705228.

[12] Izawa K-.I. and T. Yanagida, Phys. Lett. B393 (1997) 331 hep-ph/9608359].

[13] K. Kumekawa, T. Moroi and T. Yanagida, Prog. Theor. Phys. 92 (1994) 437 hep-ph/9405337.

[14] For a review, H.P. Nilles, Phys. Rep. 110 (1984) 1.

[15] H. Pargel and J.R. Primack, Phys. Rev. Lett. 48 (1982) 59.

[16] E.J. Copeland, A.R. Liddle, D.H. Lyth, E.D. Stewart and D. Wands, Phys. Rev. D49 (1994) 6410.

[17] For example, F.C Adams, J.R. Bond, K. Freese, J.A. Frieman and A.V. Olinto, Phys. Rev. D47 (1993) 426.

[18] C.L. Bennett et al., Astrophys. J. 464 (1996) L1.

[19] C. Panagiotakopolous, hep-ph/9702433;

A. Linde and A Riotto, hep-ph/9703209.

[20] M. Dine, L. Randall and S. Thomas, Phys. Rev. Lett. 75 (1995) 398 hep$\mathrm{ph} / 9503303$;

M.K. Gaillard, H. Murayama and K.A. Olive, Phys. Lett. B355 (1995) 71 hep-ph/9504307. 
[21] G. Dvali, Q. Shafi and R.K. Shaefer, Phys. Rev. Lett. 73 (1994) 1886.

[22] K. Enquvist, K.W. Ng and K.A. Olive, Nucl. Phys. B303(1988) 713.

[23] For a review, K. Intriligator and N. Seiberg, Nucl. Phys. Proc. Suppl. 45BC (1996) 1.

[24] Izawa K.-I. and T. Yanagida, Prog. Theor. Phys. 95 (1996) 829 thepth/9602180];

K. Intriligator and S. Thomas, Nucl.Phys. B473 (1996) 121 [hep-th/9603158].

[25] M. Dine and A. Riotto, hep-ph/9705386. 